Submitted to Electronics Letters, 6. FEB 1998. 1609 IEE

Optimised square passband fibre Bragg grating filter with in-band flat group delay response

Morten Ibsen, Michael K. Durkin, Martin J. Cole and Richard I. Laming.

Optoelectronics Research Centre, University of Southampton; SO17 1BJ Southampton, United Kingdom.

E-mail: mi@orc.soton.ac.uk, Tel: +441703593138

Fax: +44 1703593142

Abstract - Through a controlled apodised sinc-shaped refractive index profile we demonstrate an optimised square reflection band Bragg grating filter. The filter is $5 \mathrm{~cm}$ long and has a flat dispersion profile within the photonic bandgap and an edge steepness of $7.9 \mathrm{~dB} / \mathrm{GHz}$.

Indexing terms - Fibre Bragg gratings, square filters, WDM technology. 


\section{Optimised square passband fibre Bragg grating filter with in-band flat group delay response}

Morten Ibsen, Michael K. Durkin, Martin J. Cole and Richard I. Laming.

Optoelectronics Research Centre, University of Southampton, SO17 1BJ Southampton, United Kingdom.

E-mail:mi@orc.soton.ac.uk, Tel: +44 1703593138

Fax: +44 1703593142

Introduction:

Since the discovery of the photoinduced fibre Bragg grating [1] its importance has been acknowledged on numerous occasions and a large range of fibre Bragg grating based devices manufactured. These include chirped fibre Bragg gratings for dispersion compensation, fibre DFB lasers and very complex profiled superstructures [2-4]. There are many obvious advantages in using all-fibre devices in telecommunication networks and sensor applications, due to fibre compatibility and thereby reduced loss. As the techniques for producing fibre Bragg gratings improve, more complex structures can be manufactured.

In this paper we demonstrate a fibre Bragg grating square filter with improved flatness and wall steepness over previously reported square filters. The grating is $5 \mathrm{~cm}$ long has a bandwidth of $0.35 \mathrm{~nm}$ a reflectivity of $\sim 50 \%$ and a wall steepness of 7.9 
$\mathrm{dB} / \mathrm{GHz}$. The dispersion characteristic of the grating in the stopband is shown to be significantly lower than that of a uniform apodised grating with a similar flat-top and edge steepness criteria.

Theoretical background and design of filter:

Fourier theory is applicable to grating responses when weak gratings $(\mathrm{R} \leq \sim 50 \%)$ are considered. This predicts a sinc-shaped refractive index profile should result in a square overall reflection spectrum as has indeed been demonstrated previously $[5,6]$. Because the sinc function extends infinitely on either side of the maximum index modulation, this is impossible to realise in a Bragg grating of finite length. Therefore it is necessary to truncate the structure after a given number of side lobes. Because of this "incomplete" sinc shape, a phenomenon referred to as "Gibbs phenomenon" occurs on the edges of the stopband. In the case of the grating this gives rise to an increased reflection close to the stopband. If the grating is made longer for a given bandwidth the filter is going to become more square, i.e steeper edges and more flat-topped. Alternatively if the sinc-shaped refractive index profile is also apodised the amplitude of the side loops is reduced faster than the pure sinc, and hence the effect of a longer grating structure is obtained. This will result in a more flat-top structure, with the only disadvantage being a slightly reduced wall steepness. A gaussian apodisation profile is considered and an increased degree of apodisation is achieved by increasing the value of $a$ in (1)

$$
\delta n(z)=\delta n_{\max } \cdot \frac{\sin (p \cdot \pi \cdot z)}{p \cdot \pi \cdot z} \cdot e^{-(a \cdot z)^{2}}
$$


where $p$ is an integer determining the number of side lobes on either side of the maximum refractive index modulation, $\delta n_{\max }$, and $\mathrm{z}$ is normalised to the range $-0.5 \leq z$ $\leq 0.5$. Fig. 1 shows the resultant apodised sinc refractive index profile and the apodisation multiplier used to generate the grating spectrum. In this case $p$ is 20 giving 9 side lobes and $a$ is 2.5 .

Fabrication technique:

The grating is made using a recently developed continuous grating writing technique [7]. This technique effectively writes grating plane by grating plane and provided that the refractive index change is in the linear regime with fluence, full control of the apodisation is obtained by dephasing one grating plane with respect to the previous one [8]. A deuterium loaded fibre with an NA $\sim 0.2$ is used and an intra-cavity frequency doubled Ar-ion producing $100 \mathrm{~mW} \mathrm{CW}$ power at $244 \mathrm{~nm}$ is employed as UV source. In order to obtain the regions of periodic positive and "negative" refractive index a discrete phase-shift is inserted after each period of the sinc envelope.

\section{Results and discussion:}

Fig. 2 shows the measured reflection and time delay characteristics of a $5 \mathrm{~cm}$ long grating that has 9 lobes on either side of the maximum refractive index modulation. The overall apodisation used is the gaussian profile shown in (1) with $a=2.5$. The maximum refractive index modulation is $2.2 \cdot 10^{-4}$ resulting in $\sim 3 \mathrm{~dB}$ transmission loss through the grating. The bandwidth is $44 \mathrm{GHz}(0.35 \mathrm{~nm})$ and the wall steepness or slope at $-10 \mathrm{~dB}$ is $7.9 \mathrm{~dB} / \mathrm{GHz}$. The maximum ripple in the dispersion characteristics of the grating is periodic with $25 \mathrm{ps}$ peak to peak amplitude and a period of $30 \mathrm{pm}$, but more 
importantly the average dispersion is zero.

In comparising, Fig. 3 shows the calculated reflection and time delay characteristics of a uniform apodised grating designed to have the same edge steepness as the apodised square filter. This uniform grating is $5 \mathrm{~cm}$ long is gaussian apodised and has a peak index modulation of $3.7 \cdot 10^{-4}$. The generated time delay spectrum shows that the dispersion is not zero in-band but has an amplitude of $300 \mathrm{ps.}$

These new filters are of interest to the telecommunication industry because of their apparent square filter and flat dispersion characteristics. Problems with the dispersion close to the edge of a photonic bandgap have been pointed out when using both the grating in the stopband as a rejection filter [9] and many filters in series for multiplexed systems [10]. We show that although flat-top and steep edge gratings are possible to manufacture using more traditional grating structures it is at the expense of a non uniform in-band time delay. The sinc modulated grating however will allow tuning of the transmitter over allmost all of the stopband.

\section{Conclusion:}

We have discussed and demonstrated an optimised fibre Bragg grating square filter design. We show a $5 \mathrm{~cm}$ long grating of strength $\sim 50 \%$ and with a FWHM bandwidth of $44 \mathrm{GHz}$ and a $7.9 \mathrm{~dB} / \mathrm{GHz}$ edge steepness. The grating consists of an gaussian apodised truncated sinc-shaped refractive index profile and its reflectivity and time delay responses are shown to match theory completely. 
Acknowledgments:

The authors wish to acknowledge H. Geiger for useful discussions. This work was in part supported by Pirelli Cavi SpA. R. I. Laming acknowledges the Royal Society for the provision of a Research Fellowship. The ORC is an EPSRC funded interdisplinary research centre. 
References:

[1] MELTZ, G. and MOREY, W. W. and GLENN, W. H.: 'Formation of Bragg gratings in optical fibres by a transverse holographic method', Optics Lett., 14, pp. 823-825, 1989.

[2] DONG, L., COLE, M. J., ELLIS, A. D., DURKIN, M., IBSEN, M., GUSMEROLI, V., and LAMING, R. I.: ' $40 \mathrm{Gbit} / \mathrm{s} 1.55 \mu \mathrm{m}$ transmission over $109 \mathrm{~km}$ of non-dispersion shifted fibre with long continuously chirped fibre gratings', In proceedings to $O F C$ '97, paper PD6-1, Dallas, USA, 1997.

[3] KRINGLEBOTN, J. T., ARCHAMBAULT, J. -L., REEKIE, L. and PAYNE, D. N.: ' $\mathrm{Er}^{3+}: \mathrm{Yb}^{3-}$ co-doped fiber DFB laser', Optics. Lett., 19, pp. 2101-2103, 1994.

[4] IBSEN, M., DURKIN, M. K, COLE, M. J. and LAMING, R. I.: 'Sinc-sampled fibre Bragg grating for identical multiwavelength operation', In proceedings to OFC ' 98 , paper TuAt, San Jose, USA, 1998.

[5] STORØY, H., ENGAN, H. E., SAHLGREN, B. and STUBBE, R.: 'Position weighting of fibre Bragg gratings for bandpass filtering', Optics Lett., 1997, 22, (11), pp. 784786.

[6] UETSUKA, H., SUZUKI, R., TAMURA, K., OKANO, H., KASHIMURA, S. and HONGO, A.: 'Low reflection photoinduced Bragg grating with flat top spectral response', In proceedings to ECOC'97, 1, pp. 199-202, Edinburgh, UK., 1997.

[7] COLE, M. J., LAMING, R. I., ZERVAS, M. N., DURKIN, M. K. and IBSEN, M.: 'Continuous, arbitrary profile fibre Bragg grating fabrication technique', Paper in preparation.

[8] COLE, M. J., LOH, W. H., LAMING, R. I., ZERVAS, M. N. and BARCELOS, S.: 'Moving fibre/phase mask scanning beam technique for enhanced flexibility in producing fibre gratings with a uniform phase mask', Electron. Lett., 1995, 31, (12), pp. 1488-1489.

[9] NYKOLAK, G., LENZ, G., EGGLETON, B. J. and STRASSER, T. A.: 'Impact of fiber grating dispersion on WDM system performance', In proceedings to $O F C$ ' 98 , paper TuA3, San Jose, USA, 1998.

[10] EgGleton, B. J., LENZ, G., LiTCHINITSER, N., PATTERSON, D. B. and SLUSHER, R.E.: 'Implications of fiber grating dispersion for WDM communication systems', Photon. Technol. Lett., 9, (10), pp. 1403-1405, 1997. 
Figure captions:

Fig. 1 Refractive index profile (solid) and the apodisation multiplier (dotted) in the optimised square filter fibre Bragg grating.

Fig. 2 Measured reflection and time delay characteristics of a $5 \mathrm{~cm}$ long apodised sinc modulated square filter.

Fig. 3 Calculated reflection and time delay characteristics from a uniform gaussian apodised grating of length $5 \mathrm{~cm}$. 


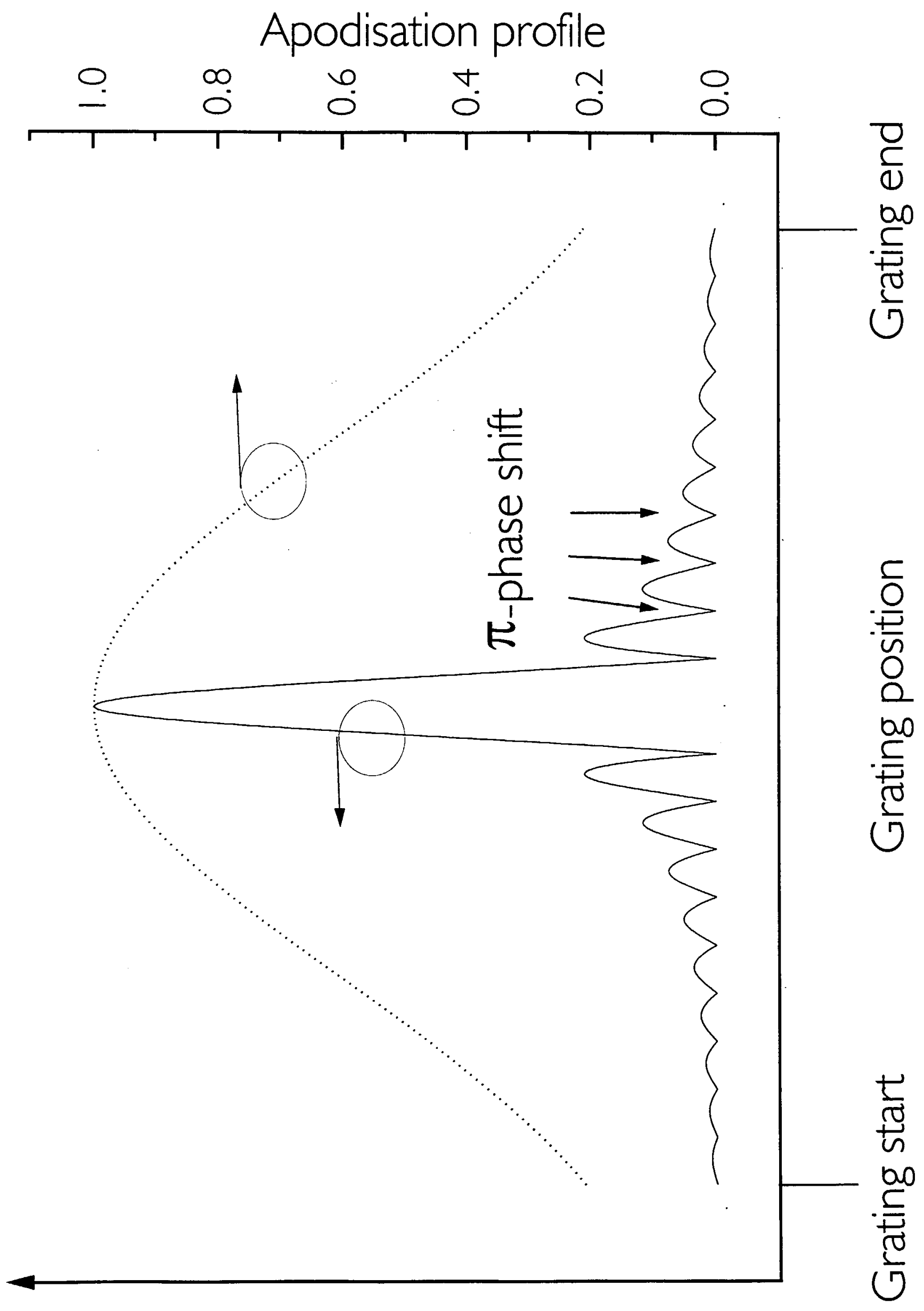

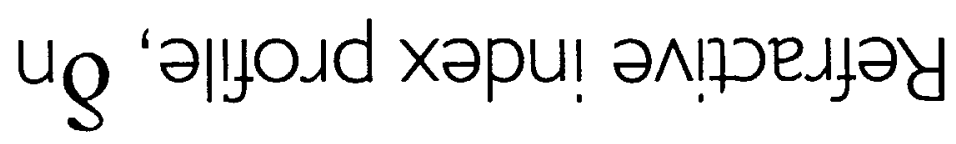




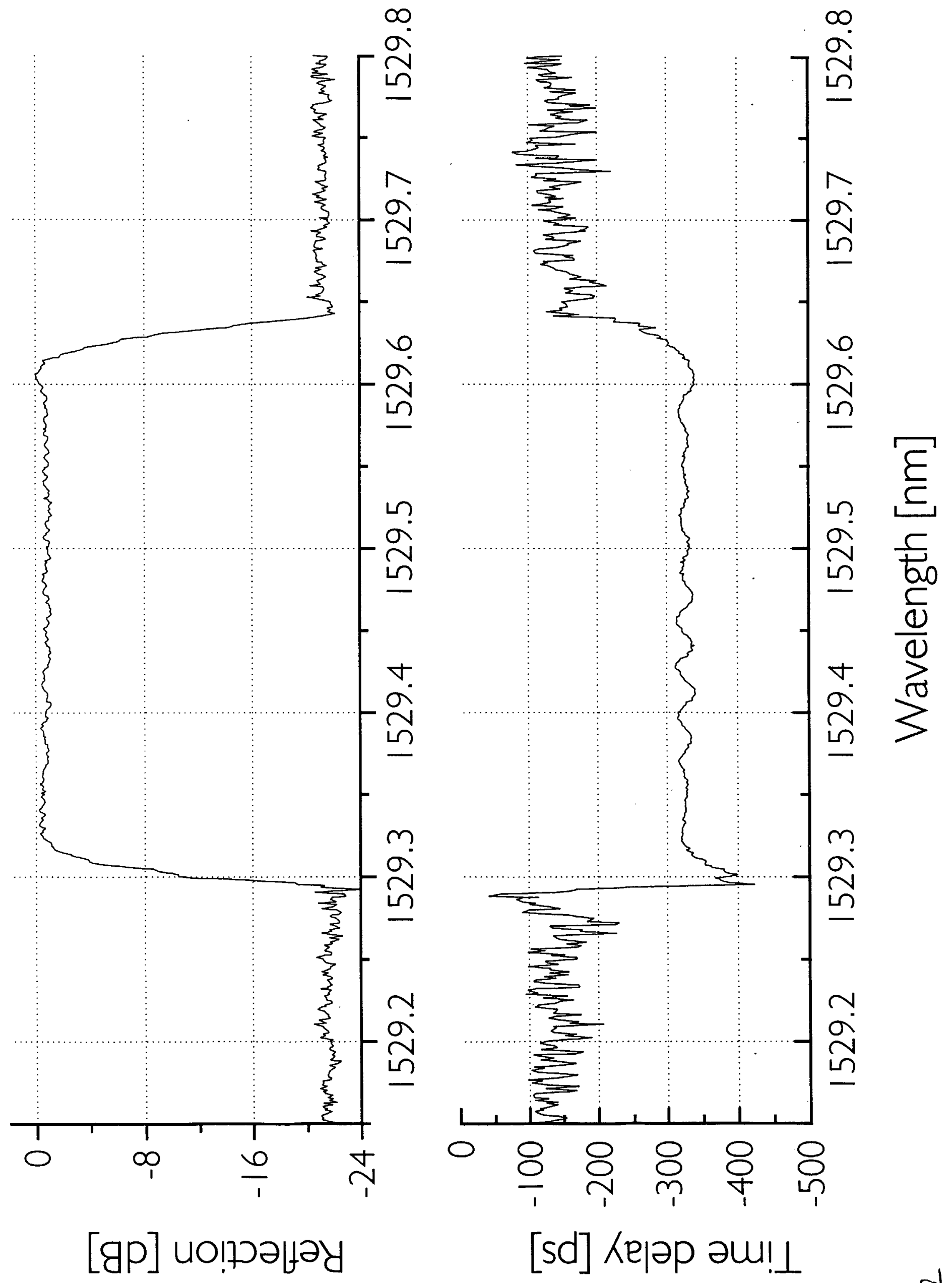




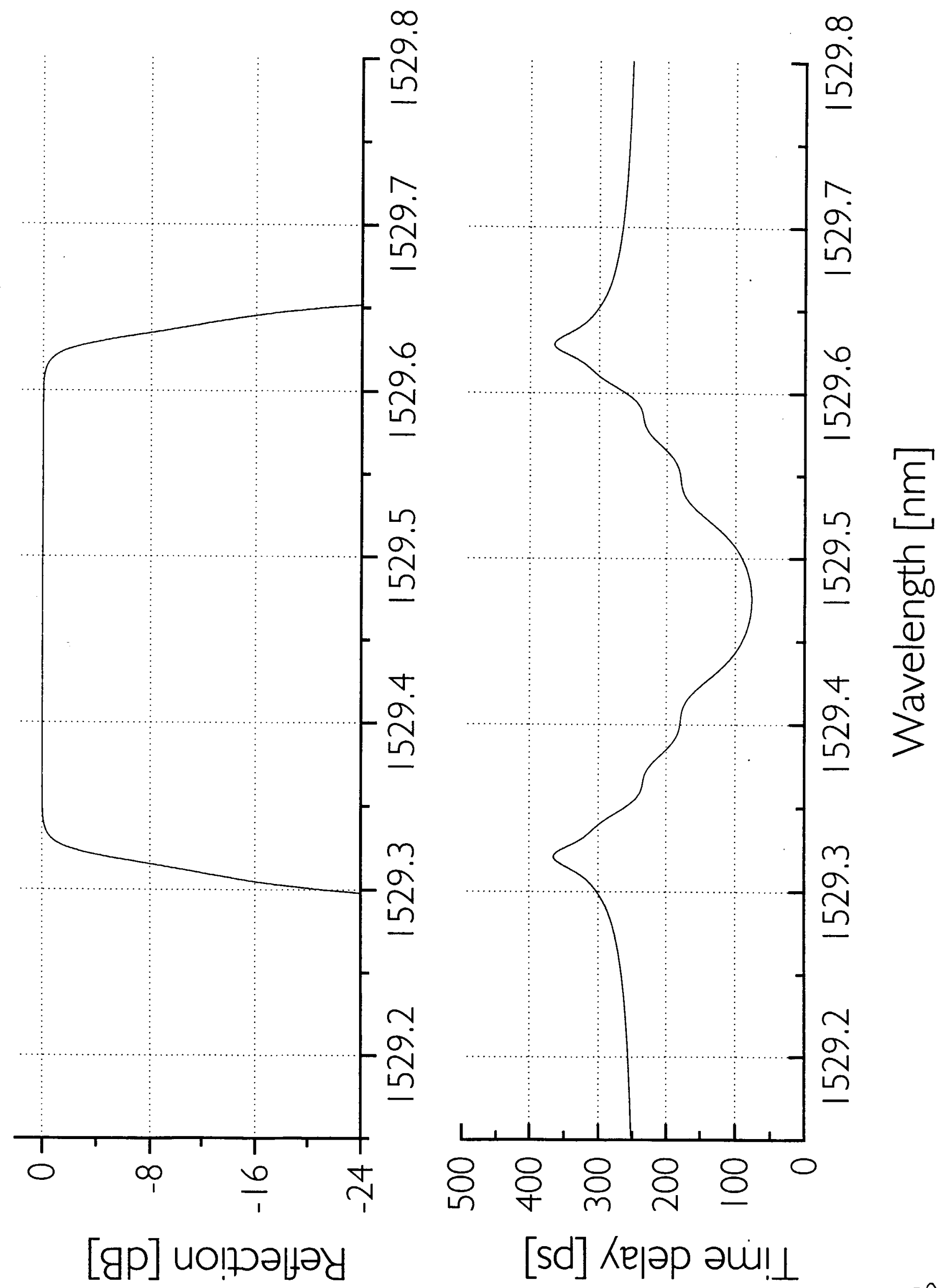

